\title{
Mother's satisfaction with the existing labor and delivery care services at public health facilities in West Shewa zone, Oromia region, Ethiopia
}

Gizachew Abdissa Bulto ${ }^{1^{*}}$, Dereje Bayissa Demissie ${ }^{2}$, Tefera Likasa Tasu ${ }^{3}$ and Getu Alemu Demisse ${ }^{4}$

\begin{abstract}
Background: Mothers' satisfaction with care during childbirth is indicators of the quality care which affects skilled birth attendance. Negative client's experiences at health facilities cause them to delay or avoid seeking care, which highlights services providers should consider and act on the expectations and experiences of women and their families. Though there are few studies conducted in Ethiopia on maternal satisfaction with Labor and Delivery (LAD) services, there is no study conducted in the study area. Therefore the study aims to assess the mother's satisfaction with existing LAD services and associated factors at all levels of health care in the West Shewa zone.

Methods: An institution-based cross-sectional study was conducted at public health facilities in West Shewa zone, Central Ethiopia. A systematic sampling technique was used to select 560 respondents by using their delivery registration number and data were collected through face to face interview. Mothers were considered satisfied if they responded satisfied/very satisfied with $75 \%$ or more of the questions assessing satisfaction. Binary and multivariable logistic regression analysis was used to identify associated factors.

Results: The overall proportion of mothers who were satisfied with the current LAD care services were $60.8 \%$. The main areas of dissatisfaction were; accessibility and cleanness of toilets/shower $72.6 \%$, overall cleanness of the facility/including waiting-area $40.1 \%$ and presence of support a person during birth 38.0\%. The presence of cultural practices $(A O R=2.5)$, discussion on the place of delivery with health worker during ANC (AOR $=1.75)$, providers asks for consent before procedure ( $A O R=2.77)$, encouraging companion to remain with mother $(A O R=2.22)$, never leave mother alone or unattended $(A O R=2.56)$, giving periodic updates on status and progress of labor $(A O R=$ 2.04) and explaining what is being done and to expect during $L A D(A O R=2.20)$ were factors identified to be significantly associated with satisfaction on LAD services.

(Continued on next page)
\end{abstract}

\footnotetext{
* Correspondence: gizachab@yahoo.com

'Department of Midwifery, College of Medicine and Health Sciences, Ambo University, Ambo, Ethiopia

Full list of author information is available at the end of the article
}

C C The Author(s). 2020 Open Access This article is licensed under a Creative Commons Attribution 4.0 International License, which permits use, sharing, adaptation, distribution and reproduction in any medium or format, as long as you give appropriate credit to the original author(s) and the source, provide a link to the Creative Commons licence, and indicate if changes were made. The images or other third party material in this article are included in the article's Creative Commons licence, unless indicated otherwise in a credit line to the material. If material is not included in the article's Creative Commons licence and your intended use is not permitted by statutory regulation or exceeds the permitted use, you will need to obtain permission directly from the copyright holder. To view a copy of this licence, visit http://creativecommons.org/licenses/by/4.0/ The Creative Commons Public Domain Dedication waiver (http://creativecommons.org/publicdomain/zero/1.0/) applies to the data made available in this article, unless otherwise stated in a credit line to the data. 
(Continued from previous page)

Conclusion: The overall satisfaction of mothers with LAD services at public health facilities in the West-Shewa zone was relatively low. Presence of cultural practices, discussion on the place of delivery, asking for consent before the procedure, encouraging companion to remain with mothers and explaining what is being done were factors identified. Therefore, all stakeholders have to emphatically work on those identified factors to improve mothers' satisfaction with LAD services.

Keywords: Mother's satisfaction, Labor and delivery, Services, Public health facilities

\section{Background}

Globally, though there is progress on maternal mortality, an estimated 295,000 maternal deaths occurred in 2017, with an overall Maternal Mortality Ratio (MMR) of 462 in low-income countries versus 11 per 100,000 live births in high-income countries. The vast majority of deaths (94\%) occurred in low-resource settings and most could have been prevented. Sub-Saharan Africa alone accounted for roughly two-thirds $(196,000)$ of maternal deaths. Ethiopia is among the fifteen countries which were identified as very high or high alert areas of global maternal deaths by world health organization [1-3]. In Ethiopia, there has been a minimal change in MMR since 2000 [4]. According to Ethiopian demographic and health survey 2016, MMR dropped from 676 in 2011 to 412 deaths per 100,000 live births in 2016 [5].

The time of birth is critical to the survival of women and their babies [6]. Provision of good quality care during pregnancy and childbirth were among the global key intervention strategies to reduce maternal and newborn mortality. United Nations estimated that the provision of effective care for all women at the time of birth in facilities could prevent 113,000 maternal deaths by 2020 [7]. Though the institutional delivery service utilization in Ethiopia is increasing, it has remained low; $10 \%$ in 2011 and $26 \%$ in 2016 [5].

Studies suggested that providing culturally appropriate maternity care services mainly have positive effects on the use of skilled maternity care [8]. In the past interventions mainly have targeted barriers to services such as accessibility and distance to health facilities, formal or informal service charges, and lack of awareness on the benefits of skilled maternity care [2, 9-13]. Despite these interventions, many women in low and middle-income countries who had awareness on the benefits of skilled maternity care and with no other geographical barriers to access health facility continued to prefer delivering at home $[10,11,14]$. Different studies conducted in SubSaharan African countries identified previous negative experiences with health facilities, poor quality of care, unprofessional behavior of health workers and disrespectful care as barriers for institutional delivery services utilization [11, 12, 15-18]. Interpersonal relationships between women and health care providers were important domains of quality of care. Maternal satisfaction which is one of a quality indicator; cover all dimensions of care including; physical environment, cleanliness, availability of resources, interpersonal relationships, maintaining privacy, promptness, cognitive care, perceived provider competency, and emotional supports [19-22]. Provision of quality of maternity care services should consider and act on the expectations and experiences of women and their families [20].

Studies indicated maternal satisfaction with care provided during labor and childbirth varies in different areas; $67 \%$ in Sweden [23], 68.8\% in Italy [24] and $49.4 \%$ had optimal and $29 \%$ had adequate satisfaction in Chile $[25,26]$. From other studies done in Nepal (86\%) [27], Bheri Zonal Hospital Nepal (89.8\%) [28], Jordan 17.8\% [29], Egypt (78.5\%) [30] and 92.5\% in southern Mozambique [31] women reported as being satisfied with the care during childbirth. In Ethiopia maternal satisfaction with care during LAD ranges from $19 \%$ at St Paul Millennium Medical College Hospital to $95 \%$ among hospitals in Wolaita Zones. Different studies identified that women's satisfaction with delivery care services was affected by their sociodemographic factors, health service quality, maternal health care utilization, obstetric factors and institutional related factors [32-41].

In Ethiopia the main reasons that have been reported for not delivering at health facilities were; health facilities were not customary, distance from facility [42], belief that it is not necessary [11], women's previous negative experiences, lack of privacy, lack of decision making power, fear of going to an unfamiliar setting and quality of care were influencing women's in deciding to seek care $[43,12]$. While many interventions aim to improve access to skilled birth care, the quality of relationships with caregivers during maternity care has received less attention [44]. Efforts to increase the use of facilitybased maternity care in low-income countries are unlikely to achieve the desired gains if there is no improvement in the quality of care provided [45]. The negative patient experiences at health facilities contribute to poor health outcomes and reinforce mistrust of institutional care which causes them to delay or avoid seeking care in health facilities [46]. 
Improved overall satisfaction level and quality of care during pregnancy and delivery are one of the main concerns in low and middle-income countries like Ethiopia, where the maternal mortality rate is high and yet the skilled birth attendance is very low. Though there are studies conducted on the level of maternal satisfaction with care during childbirth and associated factors most of them were limited to a single or few referrals or primary hospitals located in cities and there is no study conducted in the area. Therefore, there is a need to conduct the study on mothers' satisfaction with care during LAD and associated factors at health facilities in West Shewa zone addressing large geographical areas and through involving rural health centers and hospitals.

\section{Methods}

\section{Study design, area and period}

The facility-based cross-sectional study design was conducted to assess the mother's satisfaction with care during labor and delivery among mothers who gave birth at health facilities in West Shewa Zone from April to June 2018. The West Shewa zone has 22 districts and is located to the west of Addis Ababa. Ambo Town which is the capital of the zone is located $114 \mathrm{~km}$ away from Addis Ababa. According to information from the zonal health office, the total population in the zone is estimated to be $2,381,079$ of which $1,214,350$ of them were females. Currently, the zone has one university referral hospital, 1 general Hospital, 5 primary hospitals, 89 health centers and 447 health posts with $98 \%$ of potential health service coverage. Only public health centers and hospitals were currently providing $24 \mathrm{~h}$ of labor and delivery services in West Shewa zone.

\section{Source population and study population}

All women who gave birth at public health facilities in West Shewa zone were source population and all women who gave birth at selected public health facilities during the data collection period and randomly selected using systematic random sampling were our study population. Women who were referred from other health facilities after they gave birth for different reasons were excluded.

\section{Sample size and sampling procedure}

The sample size for the current study was determined by using a single population proportion formula with the assumptions of $79.1 \%$ proportion of mothers who were satisfied with the care they received during their childbirth in the Gamo Gofa zone, southern Ethiopia [36], 5\% level of significance and 5\% margin of error. Considering a $10 \%$ non-response rate and design effects of 2 , the final calculated sample size was 560 .

The stratified multistage sampling technique was used with the strata of the hospital and health center to select three hospitals and nineteen health centers with a simple random sampling technique by using the lottery method. Proportional allocation of study respondents was done for each selected hospitals and health centers by reviewing the number of their deliveries attended in the previous quarter (2174 deliveries). A systematic sampling technique was used to select mothers by using their delivery registration number. Every 3rd mother who gave birth at each selected health facilities were interviewed.

\section{Variables of the study}

Mother's satisfaction with LAD care services is the dependent variable and Independent variables were; Socio-demographic (age, marital status, religion, level of education, occupation, residence, average monthly income), obstetric related (gravidity, parity, history of institutional delivery, outcomes of previous delivery), maternal health care services and utilization (ANC, length of stay in the health facility, plan to use, mode \& outcome of delivery, client-provider interactions, health facilities environment, sex of the provider, disrespect and abuses).

\section{Measurements of satisfaction with LAD care}

The questionnaire contains six questions on physical and staff accessibility, nine on interaction with providers and staff of the facility and two on the provision of respect and privacy. The questionnaire was adapted from previously done similar studies in Ethiopia, which have adopted their tools from Donabedian quality assessment framework [21, 32, 34, 36, 38] (Additional files 1). Responses were recorded with a five-point Likert scale (very dissatisfied, dissatisfied, neutral or undecided, satisfied and very satisfied) for all seventeen questions. The reliability of the used questions was checked with Cronbach's alpha, which was found to be 0.81 . Mothers were considered satisfied with the care received from health facilities if they responded satisfied or very satisfied to $75 \%$ or more of 17 questions assessing maternal satisfaction which was determined based on previous studies conducted in Ethiopia [32, 34, 36, 38, 41].

\section{Data collection tools and techniques}

The questionnaire was initially prepared in English and translated into Afan Oromo (local language) and back to English to check for its consistency with language experts. Data was collected through face to face interview by using the Afan Oromo version questionnaire, after the health care provider's decision to discharge the mothers but before they left health facilities. Data were collected in a private setting to let them respond freely for questions. Twenty-four Nurses who were not working in the selected health facilities collected data and three Masters level supervisors have conducted 
supervision during the data collection period. Training on the objectives and techniques of data collection was given for data collectors for two days one week before the actual data collection period. Pre-test of the questionnaire was done on $5 \%$ of the women who delivered at Holeta health center and Inchini hospital and necessary corrections were made accordingly. Daily supervision was conducted by supervisors during data collection.

\section{Data processing and analysis}

The questionnaires were checked for completeness, coded and entered into EPI Data version 3.1software and then exported to SPSS windows version 23 for analysis. Bivariate analysis was used primarily to check variables which have an association with the dependent variable individually. Hosmer and Lemeshow goodness of fit test was checked, and it was found to be 0.989 on the final model. Variables which were found to have an association with the dependent variable at a $P$-value of $<$ 0.2 were then entered into multivariable logistic regression for controlling the possible effect of confounders and finally the variables which have significant association were identified based on AOR, with 95\%Confidence Interval $(\mathrm{CI})$ and $P$-value $\leq 0.05$.

\section{Ethical considerations}

Ethical clearance was obtained from the research review and ethics committee of the College of Medicine and Health Sciences, Ambo University with the reference number of AU/CMHSRCS/59/2018.

\section{Results}

In this study, a total of 558 mothers fully responded, making a response rate of $99.64 \%$. The mean age of the mothers was 26.92 with a standard deviation of 5.21 years and almost half $46.6 \%$ attended at a health center. A third of them were housewives 35.5 and $26.7 \%$ of them had primary education. The majority of respondents were married 96.2\%, belongs to Oromo ethnic group $93.9 \%$ and from urban residence $69.5 \%$ (Table 1).

\section{Obstetric characteristics of mothers who gave birth in west Shewa zone}

In this study majority of mothers were multiparas $73.7 \%$, gave birth with spontaneous vaginal delivery $79.9 \%$, gave birth without any complications $87.8 \%$ and were attended by Midwives $74.2 \%$ (Table 2).

\section{Mothers satisfaction with labor and delivery care services in west Shewa zone}

In this study, the proportion of mothers who were satisfied with the LAD care services received from health facilities in West Shewa zone was 339(60.8\%) (95\% CI: $56.5,64.9)$. Technical competency of the provider $94.1 \%$, waiting time to be seen by health worker $87.3 \%$, Health care providers (HCPs) were helpful during labor and/or birth 92.3\% and providers use of appropriate/friendly language $93.7 \%$ were those elements on which mothers were highly satisfied. Whereas the main causes of dissatisfaction identified were; accessibility and cleanness of toilets/shower $72.6 \%$, overall cleanness of the facility/including waiting-area $40.1 \%$, examination area cleanliness and comfort $35.8 \%$, presence of a support person during birth $38.0 \%$ and delivery position of choice $49.6 \%$ of them were dissatisfied (Tables 3 and 4).

\section{Factors associated with mothers' satisfaction with care during labor and delivery}

On bivariate analysis outcome of delivery, provision of comfort/pain relief as necessary, asking for consent before the procedure, presence of cultural practices in the health facilities, speaking politely to women and companion, encouraging a companion to remain with women, time or shift of delivery, discussion on the place of delivery with partner, never insulted or intimidated, explain what is being done and expect throughout LAD, giving periodic updates on status and progress, admitted to maternity waiting home before labor starts, no disrespect based on specific attribute, never leave women alone or unattended and discussing the place of delivery with health care provider during ANC follow-ups were associated with mothers satisfaction on care during LAD at $P$-value of $\leq 0.2$.

On multivariable logistic regression analysis presence of cultural practices, discussion on the place of delivery with health worker during ANC, asked for consent before the procedure, encouraging companion to remain with mother's, never leave mother alone or unattended, giving periodic updates on status and progress of labor and explaining what is being done and to expect during LAD were factors identified to be significantly associated with mothers' satisfaction with care during LAD.

This study identified that those mothers who gave birth at facilities where there are cultural practices like coffee and porridge were 2.5 times more likely to be satisfied than those where there is no $(\mathrm{AOR}=2.5,95 \% \mathrm{CI}$ : $1.28,4.94)$ and those who had discussed on the place of delivery with health worker during ANC were almost two times more likely to be satisfied than those who did not $(\mathrm{AOR}=1.75,95 \% \mathrm{CI}: 1.03,2.96)$.

The current study also identified that those mothers who were asked for consent before performing the procedure and whom no procedure was done were 2.77 and 1.9 times more likely to be satisfied with LAD service provided than those who had the procedure done without their consent $(\mathrm{AOR}=2.77,95 \% \mathrm{CI}: 1.43,5.35)$ and $(\mathrm{AOR}=1.90,95 \% \mathrm{CI}: 1.01,3.57)$ respectively. 
Table 1 A Socio-Demographic characteristic of mothers who gave birth at public health facilities in west Shewa zone, Oromia region, Ethiopia, 2018

\begin{tabular}{|c|c|c|c|}
\hline Characteristics & Categories & Number $(\boldsymbol{N}=558)$ & Percent (\%) \\
\hline \multirow[t]{3}{*}{ Type of Institution } & Health centers & 260 & 46.6 \\
\hline & Primary Hospitals & 153 & 27.4 \\
\hline & General Hospital & 145 & 26.0 \\
\hline \multirow[t]{3}{*}{ Age in years } & $17-24$ & 189 & 33.9 \\
\hline & $25-29$ & 202 & 36.2 \\
\hline & More than 29 & 167 & 29.9 \\
\hline \multirow[t]{2}{*}{ Marital Status } & Married & 537 & 96.2 \\
\hline & Other marital Status $^{1}$ & 21 & 3.8 \\
\hline \multirow[t]{3}{*}{ Religion } & Orthodox & 218 & 39.1 \\
\hline & Protestant & 301 & 53.9 \\
\hline & Other Religion ${ }^{2}$ & 39 & 7.0 \\
\hline \multirow[t]{2}{*}{ Ethnicity } & Oromo & 524 & 93.9 \\
\hline & Other Ethnicity ${ }^{3}$ & 34 & 6.1 \\
\hline \multirow[t]{4}{*}{ Educational Status } & Have no formal education & 143 & 25.6 \\
\hline & Primary education & 149 & 26.7 \\
\hline & Secondary Education & 130 & 23.3 \\
\hline & Collage and above & 136 & 24.4 \\
\hline \multirow[t]{6}{*}{ Mothers Occupation } & Government Employee & 122 & 21.9 \\
\hline & Housewife & 198 & 35.5 \\
\hline & Farmers & 84 & 15.1 \\
\hline & Merchant & 75 & 13.4 \\
\hline & Private Employee' & 66 & 11.8 \\
\hline & Other Occupation ${ }^{4}$ & 13 & 2.3 \\
\hline \multirow[t]{2}{*}{ Mothers Residence } & Urban & 388 & 69.5 \\
\hline & Rural & 170 & 30.5 \\
\hline \multirow[t]{5}{*}{ Average Monthly Income (Interquartile range) with Ethiopian Birr } & Less than 1000 & 140 & 25.1 \\
\hline & $1001-2000$ & 150 & 26.9 \\
\hline & $2001-3500$ & 132 & 23.7 \\
\hline & Greater than 3500 & 136 & 24.4 \\
\hline & Median $=2000.00$ & & \\
\hline
\end{tabular}

Keys: ${ }^{1}$ Divorced, widowed \& single, ${ }^{2}$ Wakefata \& Catholic, ${ }^{3}$ Gurage \& Tigre, ${ }^{4}$ Student \& Daily laborers

In this study, those mothers who gave birth at areas where the health workers encourage a companion to remain with them were twice more likely to be satisfied with the care received during LAD than those who were not $(\mathrm{AOR}=2.22,95 \% \mathrm{CI}: 1.42,3.46)$. Those mothers who received periodic updates on the status and progress of their labor from care providers during LAD were twice more likely to be satisfied than those who were not $(\mathrm{AOR}=2.04,95 \% \mathrm{CI}: 1.30,3.20)$ (Table 5).

\section{Discussion}

Maternal satisfaction includes all dimensions of care including; physical environment, cleanliness, availability of resources, interpersonal behavior, privacy, promptness, cognitive care, perceived provider competency, and emotional supports. In this study, the proportion of mothers who were satisfied with the LAD care services they received from health facilities in West Shewa zone was $60.8 \%$ (95\% CI: $56.5,64.9$ ). This is in-line with the study done at Referral hospitals in Amhara region (61.9\%) [32].

This finding is higher than studies conducted at Health facilities in Mizan Aman Town among mothers who gave birth in the last one year (30.4\%) [37], St. Paul's Hospital Millennium Medical College Addis Ababa (19\%) [39] and Jordan 17.8\% [29]. The possible reason for this might be due to the difference in a study setting in which the current study was conducted at 
Table 2 Obstetric characteristics of mothers who gave birth at public health facilities in west Shewa zone, Oromia region, Ethiopia, 2018

\begin{tabular}{|c|c|c|c|}
\hline Characteristics & Categories & Number $(N=558)$ & Percent (\%) \\
\hline \multirow[t]{2}{*}{ Ever given Birth before } & Yes & 411 & 73.7 \\
\hline & No & 147 & 26.3 \\
\hline \multirow[t]{3}{*}{ Number of Parity } & Primi-para & 147 & 26.3 \\
\hline & $2-3$ & 207 & 37.1 \\
\hline & $\geq 4$ & 204 & 36.6 \\
\hline \multirow[t]{2}{*}{ Had ANC follow-ups } & Yes & 528 & 94.6 \\
\hline & No & 30 & 5.4 \\
\hline \multirow[t]{4}{*}{ Number of ANC follow-ups } & No ANC follow-up & 30 & 5.4 \\
\hline & Once to twice & 90 & 16.1 \\
\hline & Three times & 173 & 31.0 \\
\hline & Four and above & 265 & 47.5 \\
\hline \multirow[t]{2}{*}{ HCPs discussed the place of delivery during ANC } & Yes & 453 & 81.2 \\
\hline & No & 105 & 18.8 \\
\hline \multirow[t]{2}{*}{ Discussed place of delivery with partner } & Yes & 400 & 71.7 \\
\hline & No & 158 & 28.3 \\
\hline \multirow[t]{2}{*}{ Duration spent on labor } & Less than or equal to $12 \mathrm{~h}$ & 410 & 73.5 \\
\hline & More than $12 \mathrm{~h}$ & 148 & 26.5 \\
\hline \multirow[t]{3}{*}{ Mode of Delivery } & Spontaneous vaginal delivery & 446 & 79.9 \\
\hline & Assisted vaginal Delivery & 44 & 7.9 \\
\hline & Cesarean section & 68 & 12.2 \\
\hline \multirow[t]{2}{*}{ Condition of the mother during childbirth } & No Complications & 490 & 87.8 \\
\hline & Had Complications & 68 & 12.2 \\
\hline \multirow[t]{2}{*}{ Time of delivery } & Day time & 298 & 53.4 \\
\hline & Night Time & 260 & 46.6 \\
\hline \multirow[t]{3}{*}{ Who attended your delivery } & Midwife & 414 & 74.2 \\
\hline & Nurse or Health Officer & 38 & 6.8 \\
\hline & Doctors or Emergency Surgeon & 106 & 19.0 \\
\hline \multirow[t]{3}{*}{ Asked your consent before performing procedures } & Not asked me & 79 & 14.2 \\
\hline & Yes & 235 & 42.1 \\
\hline & No Procedure is done for me & 244 & 43.7 \\
\hline \multirow[t]{2}{*}{ Presence of cultural practices for mothers (Coffee or porridge) } & Yes & 504 & 90.3 \\
\hline & No & 54 & 9.7 \\
\hline \multirow[t]{2}{*}{ Admitted to maternity waiting home before labor started } & Yes & 32 & 5.7 \\
\hline & No & 526 & 94.3 \\
\hline
\end{tabular}

health centers and hospitals located in small towns and rural parts of the country where the clients' awareness and expectations of health services provided were low compared to those from a big city like Addis Ababa. Also, evidence indicates mother's satisfaction with care during childbirth was lowered with time, which is in the case of Mizan Aman [37] and Jordan's [29]; and St. Paul's Hospital is a specialized teaching hospital which has many caseloads than institutions in the current study area.
The current study finding is lower than studies done at Felege Hiwot Referral Hospital Bahir Dar (74.9\%) [33], Debre Markos among mothers who gave birth within one year (81.7\%) [34], Asrade Swede Memorial Primary Hospital West Gojjam (88\%) [35], Hospitals in Wolaita Zone (95\%) [47], Gamo Gofa Zone (79.1\%) [36], Harari region (84.7\%) [38], Asella hospital (80.7\%) [40], health facilities in West Arsi Zone (74.6\%) [41], Southern Mozambique (92.5\%) [31] and Bheri Zonal Hospital, Nepal (89.88\%) [28]. These variations might be due to 
Table 3 Mother's satisfaction with care during labor and delivery at public health facilities in West Shewa Zone, Oromia region, Ethiopia, 2018

\begin{tabular}{|c|c|c|c|}
\hline \multirow[t]{2}{*}{ Categories } & \multirow[t]{2}{*}{ Variables } & \multicolumn{2}{|c|}{ Satisfied with LAD care } \\
\hline & & $\begin{array}{l}\text { Yes } \\
\boldsymbol{N}(\%)\end{array}$ & $\begin{array}{l}\mathrm{No} \\
\boldsymbol{N}(\%)\end{array}$ \\
\hline \multirow[t]{6}{*}{ Physical and Staff accessibility } & Examination area cleanliness and comfort & $358(64.2)$ & 200(35.8) \\
\hline & Overall cleanness of the facility/including waiting area & 334(59.9) & $224(40.1)$ \\
\hline & Accessibility and cleanness of toilets/shower & 153(27.4) & 405(72.6) \\
\hline & Availability of supplies basic drugs and equipment & $429(76.9)$ & $129(23.1)$ \\
\hline & Technical competency of care providers & $525(94.1)$ & $33(5.9)$ \\
\hline & Waiting time to be seen by the health worker & $487(87.3)$ & $71(12.7)$ \\
\hline \multirow[t]{9}{*}{ Interaction with Providers and Staff of the Health Facility } & $\mathrm{HCP}$ were friendly and welcoming on arrival & $477(85.5)$ & $81(14.5)$ \\
\hline & HCPs were encouraging and reassuring during $L A D$ & 457(81.9) & $101(18.1)$ \\
\hline & HCPs were helpful during labor and/or birth & $515(92.3)$ & $43(7.7)$ \\
\hline & Overall care during labor and birth was good & $505(90.5)$ & $53(9.5)$ \\
\hline & HCP kept you informed what was happening during LAD & $351(62.9)$ & 207(37.1) \\
\hline & Involvement of patient in decision making & 472(84.6) & $86(15.4)$ \\
\hline & Presence of support person during birth & $346(62.0)$ & 212(38.0) \\
\hline & Confidentiality and trust in providers & 458(82.1) & 100(17.9) \\
\hline & Delivery position of patient choice & $281(50.4)$ & 277(49.6) \\
\hline \multirow[t]{2}{*}{ Provision of Respect and Privacy } & Respect and assurance of privacy during exam and delivery & 489(87.6) & $69(12.4)$ \\
\hline & Providers used appropriate/friendly language & 523(93.7) & $35(6.3)$ \\
\hline \multicolumn{2}{|c|}{ Overall satisfaction with labor and delivery care services received from health facilities in West Shewa zone } & $339(60.8 \%)$ & 219(39.2) \\
\hline
\end{tabular}

most of those studies were conducted at hospitals where there are relatively adequate infrastructure, supplies and human resources with the presence of a mix of professionals, including specialty care in those study areas, but the current study was conducted at health centers and hospitals located more in small towns and in rural parts of the country. It is also due to the differences in the quality of services provided in those study areas specifically Felege Hiwot, Asella, Wolaita and hospitals in Harar. Studies in Wolaita and Harar also included private hospitals that were providing quality services than government institutions.

Health centers and hospitals working environment is the main source of dissatisfaction in the study area. The main areas of dissatisfaction in the current study were; accessibility and cleanness of toilets or shower rooms, overall cleanness of the facility/including waiting area, examination area cleanliness, and comfort, presence of a support person during birth and delivery position of choice. In line with these previous studies also indicated; overall cleanliness and comfort of examination rooms and waiting areas, cleanliness and access to the toilets, and availability of hand-washing facility and shower were the main areas of dissatisfaction [35, 36, 40, 41, 47].

The provision of culturally sensitive quality health services is important for enhancing maternal health services utilization. Those mothers who gave birth at facilities where there is a cultural practice like coffee and porridge were 2.5 times more likely to be satisfied than those where there are no practices. The reason might be due to those practices that were common to do in the area immediately after the mother gave birth at home, therefore, those mothers who get an opportunity to practice were more likely to feel the health institutions like their home.

Those who had discussed the place of delivery with health workers during ANC were 1.75 times more likely to be satisfied than those who did not. In line with this, a study done at Debre Markos showed that having a plan to deliver at health institutions was 3 times more likely to be satisfied than those who did not [34]. This might be due to those mothers who had discussed the place of delivery during $\mathrm{ANC}$ were likely to receive education on birth preparedness and complication readiness plan and at some institutions providers let the pregnant mother visit their LAD room to familiarize them.

Requesting for consent before any procedure is found to be an important factor affecting maternal satisfaction with LAD care services. Those women who were asked for consent before performing the procedure and those whom no procedure was done were 2.77 and 1.9 times more likely to be satisfied with LAD service provided than those who had a procedure done without asking their consent. The possible explanation for this might be 
Table 4 Satisfaction with care during LAD with some socio-demographic and obstetric characteristics among mothers' who gave birth at public health facilities in West Shewa Zone, Oromia region, Ethiopia, 2018

\begin{tabular}{|c|c|c|c|}
\hline Characteristics & Categories & Dissatisfied with LAD care (\%) & Satisfied with LAD care (\%) \\
\hline \multirow[t]{3}{*}{ Type of Institution } & Health centers & $104(40.0)$ & $156(60.0)$ \\
\hline & Primary Hospitals & $57(37.3)$ & $96(62.7)$ \\
\hline & General Hospital & $58(40.0)$ & $87(60.0)$ \\
\hline \multirow[t]{3}{*}{ Age in years } & $17-24$ & $71(37.6)$ & $118(62.4)$ \\
\hline & $25-29$ & $80(39.6)$ & $122(60.4)$ \\
\hline & More than 29 & $68(40.7)$ & $99(59.3)$ \\
\hline \multirow[t]{2}{*}{ Marital Status } & Married & $211(39.3)$ & $326(60.7)$ \\
\hline & Other marital Status ${ }^{1}$ & $8(38.1)$ & $13(61.9)$ \\
\hline \multirow[t]{3}{*}{ Religion } & Orthodox & $70(32.1)$ & $148(67.9)$ \\
\hline & Protestant & $132(43.9)$ & $169(56.1)$ \\
\hline & Other Religion ${ }^{2}$ & $17(43.6)$ & $22(56.4)$ \\
\hline \multirow[t]{2}{*}{ Ethnicity } & Oromo & $210(40.1)$ & $314(59.9)$ \\
\hline & Other Ethnicity ${ }^{3}$ & $9(26.5 \%)$ & $25(73.5)$ \\
\hline \multirow[t]{4}{*}{ Educational Status } & Have no formal education & $60(42.0)$ & $83(58.0)$ \\
\hline & Primary education & $73(49.0)$ & $76(51.0)$ \\
\hline & Secondary Education & $41(31.5)$ & $89(68.5)$ \\
\hline & Collage and above & $45(33.1)$ & $91(66.9)$ \\
\hline \multirow[t]{6}{*}{ Mothers Occupation } & Government Employee & $41(33.6)$ & $81(66.4)$ \\
\hline & House wife & $89(44.9)$ & $109(55.1)$ \\
\hline & Farmers & $30(35.7)$ & $54(64.3)$ \\
\hline & Merchant & $27(36.0)$ & $48(64.0)$ \\
\hline & Private Employee' & $25(37.9)$ & $41(62.1)$ \\
\hline & Other Occupation ${ }^{4}$ & $7(53.8)$ & $6(46.2)$ \\
\hline \multirow[t]{2}{*}{ Mothers Residence } & Urban & $152(39.2)$ & $236(60.8)$ \\
\hline & Rural & $67(39.4)$ & $103(60.6)$ \\
\hline \multirow[t]{3}{*}{ Parity } & Primi-para & $59(40.1)$ & $88(59.9)$ \\
\hline & $2-3$ & 80 (38.6) & $127(61.4 \%)$ \\
\hline & $\geq 4$ & $80(39.2)$ & $124(60.8 \%)$ \\
\hline \multirow[t]{2}{*}{ Had ANC follow-ups } & Yes & $204(38.6)$ & $324(61.4)$ \\
\hline & No & $15(50.0)$ & $15(50.0)$ \\
\hline \multirow[t]{2}{*}{ Duration spent on labor } & Less than/equal to $12 \mathrm{~h}$ & $187(45.6)$ & $223(54.4)$ \\
\hline & More than $12 \mathrm{~h}$ & $32(21.6)$ & $116(78.4)$ \\
\hline \multirow[t]{3}{*}{ Mode of Delivery } & Spontaneous vaginal delivery & $187(41.9)$ & $259(58.1)$ \\
\hline & Assisted vaginal Delivery & $12(27.3)$ & $32(72.7)$ \\
\hline & Cesarean section & $20(29.4)$ & $48(70.6)$ \\
\hline \multirow[t]{2}{*}{ Outcomes of delivery } & Alive & $215(39.8)$ & $325(60.2 \%)$ \\
\hline & Dead & $4(22.2)$ & $14(77.8)$ \\
\hline \multirow[t]{2}{*}{ Condition of mother during childbirth } & No Complications & $198(40.4)$ & $292(59.6)$ \\
\hline & Had Complications & $21(30.9)$ & $47(69.1)$ \\
\hline \multirow[t]{2}{*}{ Time of delivery } & Day time & $101(33.9)$ & $197(66.1)$ \\
\hline & Night Time & $118(45.4)$ & $142(54.6)$ \\
\hline
\end{tabular}

Keys: ${ }^{1}$ divorced, widowed \& single, ${ }^{2}$ Wakefata \& Catholic, ${ }^{3}$ Gurage \& Tigre, ${ }^{4}$ Student \& Daily laborers 
Table 5 Factors associated with Mothers' satisfaction with care during labor and delivery at health Facilities in West Shewa Zone, Oromia region, Ethiopia, 2018

\begin{tabular}{|c|c|c|c|c|c|}
\hline \multirow[t]{2}{*}{ Variables } & \multicolumn{2}{|c|}{ Satisfied with care during LAD } & \multirow{2}{*}{$\begin{array}{l}\text { Crude OR } \\
95 \% \mathrm{Cl}\end{array}$} & \multirow{2}{*}{$\begin{array}{l}\text { Adjusted OR } \\
95 \% \mathrm{Cl}\end{array}$} & \multirow[t]{2}{*}{$\boldsymbol{P}$-value } \\
\hline & Yes & No & & & \\
\hline \multicolumn{6}{|c|}{ Presence of cultural practices (Coffee or Porridge) } \\
\hline Yes & 45(83.3) & $9(16.7)$ & $3.57(1.70,7.46)$ & $2.51(1.28,4.94)$ & \\
\hline No & 294(58.3) & $210(41.7)$ & 1 & 1 & 0.007 \\
\hline \multicolumn{6}{|c|}{ Discussed on the place of delivery during ANC } \\
\hline Yes & $301(66.4)$ & 152(33.6) & $3.49(2.24,5.43)$ & $1.75(1.03,2.96)$ & 0.036 \\
\hline No & $38(36.2)$ & $67(63.8)$ & 1 & 1 & \\
\hline \multicolumn{6}{|c|}{ Asked for consent before performing procedures } \\
\hline Not asked me & 28(35.4) & $51(64.6)$ & 1 & 1 & 0.008 \\
\hline Yes & 164(69.8) & $71(30.2)$ & $4.20(2.45,7.20)$ & $2.77(1.43,5.35)$ & 0.002 \\
\hline No procedure is done & $147(60.2)$ & 97(39.8) & $2.76(1.62,4.67)$ & $1.90(1.01,3.57)$ & 0.045 \\
\hline \multicolumn{6}{|c|}{ Encourage companion to remain with mother's } \\
\hline Yes & $271(70.9)$ & $111(29.1)$ & $3.87(2.66,5.64)$ & $2.22(1.42,3.46)$ & \\
\hline No & 68(38.6) & 108(61.4) & 1 & 1 & 0.0004 \\
\hline \multicolumn{6}{|c|}{ Never leave women alone or unattended } \\
\hline Yes & $258(74.4)$ & $89(25.6)$ & $4.65(3.22,6.71)$ & $2.56(1.61,4.06)$ & \\
\hline No & $81(38.4)$ & 130(61.6) & 1 & 1 & 0.00006 \\
\hline \multicolumn{6}{|c|}{ Gives periodic updates on status and progress of labor } \\
\hline Yes & 273(71.7) & 108(28.3) & $4.25(2.91,6.20)$ & $2.04(1.30,3.20)$ & \\
\hline No & $66(37.3)$ & $111(62.7)$ & 1 & 1 & 0.002 \\
\hline \multicolumn{6}{|c|}{ Explain what is being done and to expect during $L A D$} \\
\hline Yes & $261(75.2)$ & $86(24.6)$ & $5.17(3.57,7.49)$ & $2.20(1.39,3.47)$ & \\
\hline No & $78(37.0)$ & 133(63.0) & 1 & 1 & 0.001 \\
\hline
\end{tabular}

those mothers whom the provider did not take consent before the procedure was likely to perceive as the provider undermined them and feel as they did not consider their right for engagement in their care decision making.

Evidence suggests companionship of choice during labor and childbirth may increase spontaneous vaginal birth, reduces cesarean section and instrumental vaginal births [48]. The world health organization also recommended a companion of choice for all women throughout labor and childbirth [6]. In this study, those mothers who gave birth at institutions where the health workers encourage companion to remain with mothers were twice more likely to be satisfied with the care received during LAD than those who were not. In agreement with this study done in southern Mozambique has shown having a companion during childbirth increased the overall satisfaction of mothers [31] and in Gamo Gofa Zone the presence of support persons during childbirth was significantly associated with institutional delivery service satisfaction [36].

Women who were provided with continuous support during labor and childbirth were at increased chance of spontaneous vaginal birth, had no harm, and were more satisfied [48]. Mothers who were provided uninterrupted care during LAD from care providers were 2.56 times more likely to be satisfied with the care they received than those who were left alone or unattended. Similarly, the study done in southern Mozambique showed that mothers who experienced as being abandoned when needing help reported low levels of satisfaction [31], and in Harar Hospitals participants who waited for $15 \mathrm{~min}$ or less to be seen by the HCPs were 2.5 times more likely to be satisfied compared to women who waited for more [38].

The study revealed that those mothers whom health workers provided periodic updates on the status and progress of their labor from care providers during LAD were twice more likely to be satisfied than those who were not and those who gave birth at areas where care providers explain what is being done and to expect during LAD were twice more likely to be satisfied than those who were not provided. Likewise, studies done in Nepal indicated having an opportunity to ask questions was positively associated with client satisfaction [49], at referral Hospitals in Ethiopia inadequate information about the drugs and explanation of procedures to be 
done to the client were found to be major causes of dissatisfaction [50], St. Paul Hospital Addis Ababa shown that the women who had an opportunity to talk with health care providers were 2.44 times satisfied than those who did not [39], and at Asrade Zewude Memorial primary hospital mothers who care providers listened and answered their questions during delivery were more likely to be satisfied than those who did not [35].

\section{Limitations and strength}

The study is not free from social desirability bias since we have collected data at health facilities with an exit interview and being cross-sectional as it is difficult to assess temporal relationships were some of the limitations of this study. Addressing a larger number of health facilities both involving health centers and hospitals might be the strength of this study compared to other previous studies.

\section{Conclusion}

The study demonstrated the overall satisfaction of mothers with LAD care services they received from public health facilities in West Shewa zone was relatively low. The study identified the presence of cultural practices, discussion on place of delivery with health worker during ANC, asking for consent before the procedure, encouraging companion to remain with mother, never leaving the mother alone or unattended, giving periodic updates on status and progress of labor and explaining what is being done and to expect during LAD were factors identified to be significantly associated with mothers satisfaction on care during LAD.

Therefore, the federal ministry of health, regional health bureau, zonal health office and health facilities should have to work on making health facilities working environments more conducive or comfortable for the clients through maintaining the cleanliness of the examination and waiting-areas and making clean toilets, shower rooms accessible for the mothers in all health facilities.

Health facilities and health workers should work on promoting cultural practices in their health facilities, encourage the presence of a companion, providing uninterrupted care, requesting for consent before any procedure, and give periodic updates on the status and progress of labor during LAD is recommended. Further study is also recommended to explore on cultural preferences of mothers during labor and childbirth which might affect their delivery preference and satisfaction level.

\section{Supplementary information}

Supplementary information accompanies this paper at https://doi.org/10. 1186/s12884-020-02998-6.

Additional file 1. English version Questionnaire

\section{Abbreviations}

ANC: Ante Natal Care; AOR: Adjusted Odds Ratio; HCPs: Health care Professionals; LAD: Labor And Delivery; MMR: Maternal Mortality Ration; PNC: Post Natal Care

\section{Acknowledgements}

We are grateful to West Shewa Zone Health Offices and health institutions for their support through providing us necessary information's during proposal development and support during data collection. We also appreciate all study respondents for their contributions.

\section{Authors' contributions}

GAB conceptualized the study, designed the study instrument and conducted the data analysis and wrote the first draft and subsequent drafts of the manuscript as principal investigator. DBD TLT and GAD take part in designing the study tool, participated in data analysis and revised consequent drafts. All authors read and approved the final manuscript.

\section{Funding}

Funding for this study was obtained from Ambo University, Office of vice president for research and community services. The university was not involved in the design of the study and collection, analysis, and interpretation of data.

\section{Availability of data and materials}

Datasets used in the current study are available from the corresponding author on reasonable request.

\section{Ethics approval and consent to participate}

Ethical clearance was obtained from the research ethics review committee of the College of Medicine and Health Sciences, Ambo University. A formal letter of cooperation was written to all respective Health institutions. Written informed consent was obtained from study participants after fully explaining the nature of the study in their local languages as it is attached in the questionnaire. The collected information was kept confidential without the name of the study participants.

\section{Consent for publication}

Not applicable.

\section{Competing interests}

Authors declared that they have no competing interests.

\section{Author details}

'Department of Midwifery, College of Medicine and Health Sciences, Ambo University, Ambo, Ethiopia. 'Department of Neonatal Nursing, St. Paul's Hospital Millennium Medical College, Addis Ababa, Ethiopia. ${ }^{3}$ Department of Nursing, College of Medicine and Health Sciences, Ambo University, Ambo, Ethiopia. ${ }^{4}$ Department of Public Health, College of Medicine and Health Sciences, Ambo University, Ambo, Ethiopia.

Received: 16 January 2020 Accepted: 7 May 2020

Published online: 19 May 2020

\section{References}

1. WHO. World Health Organization: Maternal mortality key facts: 2017 [Internet]. 2019. Available from: https://www.who.int/news-room/factsheets/detail/maternal-mortality.

2. WHO. Trends in Maternal Mortality : 1990 to 2015 estimates by WHO, UNICEF, UNFPA, World Bank Group and the United Nations Population Division. Geneva: World Health Organization [Internet]. 2015; Available from: www.who.int.

3. Alkema L, Chou D, Hogan D, Zhang S, Moller A, Gemmill A, et al. Global, regional, and national levels and trends in maternal mortality between 1990 and 2015 , with scenario-based projections to 2030 : a systematic analysis by the UN Maternal Mortality Estimation Inter-Agency Group. Lancet [Internet] 2016;387(10017):462-474. Available from: http://dx.doi.org/ https://doi.org/10.1016/S0140-6736(15)00838-7.

4. Tessema GA, Laurence CO, Melaku YA, Misganaw A, Woldie SA, Hiruye A, et al. Trends and causes of maternal mortality in Ethiopia during 1990-2013: 
findings from the global burden of diseases study 2013. BMC Public Health. 2017;17(1):1-8

5. Central Statistical Agency (CSA) [Ethiopia] and ICF. Ethiopia Demographic and Health Survey 2016: Key indicators report. Addis Ababa: CSA and ICF; 2016.

6. World Health Organization. WHO recommendations: Intrapartum care for a positive childbirth experience [internet]. Geneva: World Health Organization; 2018. p. 212. Available from: http://apps.who.int/iris/bitstream/10665/2601 78/1/9789241550215-eng.pdf?ua=1\%0Ahttp://www.who.int/ reproductivehealth/publications/intrapartum-care-guidelines/en/.

7. Nation United(UN) Secretary-General. The global strategy for women's, children's and adolescents' health (2016-2030) [Internet]. New York: United Nation; 2016. Available from: www.everywomaneverychild.org.

8. Coast E, Jones E, Lattof SR, Portela A. Effectiveness of interventions to provide culturally appropriate maternity care in increasing uptake of skilled maternity care: a systematic review. Health Policy Plan. 2016;31(10):1479-91.

9. Mamdani M, Bangser M. Poor people ' s experiences of health Services in Tanzania: a literature review. Reprod Health Matters. 2004;12(24):138-53.

10. Bohren MA, Hunter EC, Munthe-Kaas HM, Souza J, Vogel JP, Gülmezoglu A. Facilitators and barriers to facility-based delivery in low- and middle-income countries: a qualitative evidence synthesis. Reprod Health. 2014;11(1):71-88.

11. Shiferaw S, Spigt M, Godefrooij M, Melkamu Y, Tekie M. Why do women prefer home births in Ethiopia ? BMC Pregnancy Childbirth. 2013;13(5):1-10.

12. Kea AZ, Tulloch O, Datiko DG, Theobald S, Kok MC. Exploring barriers to the use of formal maternal health services and priority areas for action in Sidama zone, southern Ethiopia. BMC Pregnancy Childbirth. 2018;18(1):1-12.

13. Sialubanje C, Massar K, Pijl MSG Van Der, Kirch EM, Hamer DH, Ruiter RAC. Improving access to skilled facility-based delivery services: Women 's beliefs on facilitators and barriers to the utilisation of maternity waiting homes in rural Zambia. Reprod Health [Internet]. 2015;1-13. Available from: http://dx.doi.org/https://doi.org/10.1186/s12978-015-0051-6.

14. Kruk ME, Paczkowski M, Mbaruku G, De Pinho H, Galea S. Women's Preferences for Place of Delivery in Rural Tanzania: A Population-Based Discrete Choice Experiment. Am J Public Health. 2015;99(9):1666-72.

15. Kruk ME, Paczkowski MM, Tegegn A, Tessema F, Hadley C, Asefa M, et al. Women's preferences for obstetric care in rural Ethiopia: a population-based discrete choice experiment in a region with low rates of facility delivery. J Epidemiol Community Health [Internet]. 2010;64(11):984-8 Available from: http://jech.bmj.com/content/64/11/984.abstract.

16. Kujawski S, Mbaruku G, Freedman LP, Ramsey K, Moyo W, Kruk ME. Association between disrespect and Abuse during childbirth and women's confidence in health facilities in Tanzania. Matern Child Health J. 2015; 19(10):2243-50

17. Kifle MM, Kesete HF, Gaim HT, Angosom GS, Araya MB. Health facility or home delivery? Factors influencing the choice of delivery place among mothers living in rural communities of Eritrea. J Health Popul Nutr. 2018; 37(1):22

18. Dodzo MK, Mhloyi M. Home is best: Why women in rural Zimbabwe deliver in the community. PLoS One. 2017;1-23.

19. Srivastava A, Avan BI, Rajbangshi P, Bhattacharyya S. Determinants of women's satisfaction with maternal health care: a review of literature from developing countries. BMC Pregnancy Childbirth. 2015;15:97.

20. Bohren MA, Titiloye MA, Kyaddondo D, Hunter EC, Oladapo OT, Tunçalp Ö, et al. Defining quality of care during childbirth from the perspectives of Nigerian and Ugandan women : a qualitative study. Int J Gynecol Obstet. 2017;139(December):4-16.

21. Donabedian A. An Introduction to Quality Assurance in Health Care [Internet]. Bashshur R, editor. New York: Oxford University Press, Inc.; 2003. 1-37 p. Available from: http://www.oup-usa.org.

22. World Health Organization. Standards for improving quality of maternal and newborn care in health facilities [Internet]. 20 Avenue Appia, 1211 Geneva 27, Switzerland: WHO Library Cataloguing-in-Publication Data; 2016. 40-47 p. Available from: http://www.who.int.

23. Rudman A, El-khouri B, Waldenstro U. Women 's satisfaction with intrapartum care - a pattern approach. J Adv Nurs. 2007;59(5):474-87.

24. Lazzerini M, Valente EP, Covi B, Semenzato C. Use of WHO standards to improve quality of maternal and newborn hospital care : a study collecting both mothers ' and staff perspective in a tertiary care hospital in Italy. BMJ Open Qual. 2019;8(1-15):1-13.

25. Pantoja L, Weeks FH, Ortiz J, Cavada G, Foster J, Binfa L. Dimensions of childbirth care associated with maternal satisfaction among low-risk Chilean women. Health Care Women Int [Internet]. 2020 ;41(1):89-100. Available from: https://doi.org/https://doi.org/10.1080/07399332.2019.1590360.

26. Weeks F, Pantoja L, Ortiz J, Foster J, Cavada G, Binfa L. Labor and birth care satisfaction associated with medical interventions and accompaniment during labor among Chilean women. J Midwifery \&Women's Heal. 2017;62: 196-203.

27. Paudel YR, Mehata S, Paudel D, Dariang M, Aryal KK, Poudel P, et al. Women 's satisfaction of maternity Care in Nepal and its Correlation with intended future utilization. Int J Reprod Med. 2015;2015:9.

28. Panth A, Kafle P. Maternal satisfaction on delivery service among postnatal mothers in a government hospital. Mid-Western Nepal Obstet Gynecol Int. 2018;2018:1-11.

29. Khitam ISM, Insaf S, Caroline H, Debra C. Womens satisfaction with hospitalbased intrapartum care: a Jordanian study. Int J Nurs Midwifery. 2014;6(3): 32-9.

30. Sayed W, Eldeen D, Elaal MA, Mohammed HS, Abbas AM, Zahran KM. Maternal satisfaction with delivery services at tertiary university hospital in upper Egypt, is it actually satisfying? Int J Reprod Contracept Obstet Gynecol. 2018;7(7):2547-52.

31. Mocumbi S, Högberg U, Lampa E, Sacoor C, Valá A, Bergström A, et al. Mothers ' satisfaction with care during facility-based childbirth: a crosssectional survey in southern Mozambique. BMC Pregnancy Childbirth. 2019; 19:303.

32. Tayelgn A, Zegeye DT, Kebede Y. Mothers ' satisfaction with referral hospital delivery service in Amhara Region, Ethiopia. BMC Pregnancy Childbirth [Internet]. 2011;11(1):78. Available from: http://www.biomedcentral.com/14 71-2393/11/78

33. Mekonnen ME, Yalew WA, Anteneh ZA. Women 's satisfaction with childbirth care in Felege Hiwot Referral Hospital , Bahir Dar city, Northwest Ethiopia , 2014 : cross sectional study. BMC Res Notes. 2015;8:528.

34. Bitew K, Ayichiluhm M, Yimam K. Maternal satisfaction on delivery service and its associated factors among mothers who gave birth in public health facilities of Debre Markos town, Northwest Ethiopia. Biomed Res Int. 2015; 2015:1-7.

35. Asres GD. Satisfaction and associated factors among mothers delivered at Asrade Zewude memorial primary hospital, Bure, west Gojjam, Amhara, Ethiopia: a cross sectional study. J Public Manag Res. 2018;4(1):14.

36. Tesfaye R, Worku A, Godana W, Lindtjorn B. Client Satisfaction with Delivery Care Service and Associated Factors in the Public Health Facilities of Gamo Gofa Zone, Southwest Ethiopia : In a Resource Limited Setting. Obstet Gynecol Int. 2016;2016:1-8.

37. Teklemariam Ergat $Y$, Wasihun Adraro A, Tariku R, Addisu G. Level of maternal satisfaction and its determinants at health facilities in Mizan-Aman town, Ethiopia: cross sectional study. Int J Women's Heal Wellness. 2019;5(1):4-8.

38. Getenet AB, Teji Roba K, Seyoum Endale B, Mersha Mamo A, Darghawth R. Women\&rsquo;s satisfaction with intrapartum care and its predictors at Harar hospitals, eastern Ethiopia: a cross-sectional study. Nurs Res Rev. 2018; 9:1-11.

39. Demas T, Getinet T, Bekele D, Gishu T, Birara M, Abeje Y. Women's satisfaction with intrapartum care in St Paul's hospital millennium medical college Addis Ababa Ethiopia: a cross sectional study. BMC Pregnancy Childbirth. 2017:17(1):1-8.

40. Roza A, Tafa M, Hailu F. Maternal satisfaction with the delivery Services in Assela Hospital, Arsi zone. Oromia Region Gynecol Obstet. 2014;4(12):1-8.

41. Edaso AU, Teshome GS. Mothers' Satisfation With Delivery Services and Associated Factorsat Health Institutions in West Arsi, Oromia Regional State, Ethiopia. MOJ Womens Heal. 2019;8(1):1-8.

42. CentralStatisticalAgency[Ethiopia] II. Ethiopia Demographic and Health Survey 2011 [Internet]. Addis Ababa: Central Statistical Agency and ICF International; 2012. Available from: csa@ethionet.et.

43. Shifraw T, Berhane $Y$, Gulema H, Kendall T, Austin A. A qualitative study on factors that influence women's choice of delivery in health facilities in Addis Ababa, Ethiopia. BMC Pregnancy Childbirth [Internet] 2016;16(1):1-6. Available from: http://dx.doi.org/https://doi.org/10.1186/s12884-016-1105-7.

44. WhiteRibbonAlliance(WRA). Respectful maternity care campaign update April 2015; 2015. p. 1-3. Available from: whiteribbonalliance.org.

45. Rosen HE, Lynam PF, Carr C, Reis V, Ricca J, Bazant ES. Direct observation of respectful maternity care in five countries: a cross-sectional study of health facilities in east and southern Africa. BMC Pregnancy Childbirth. 2015;15:306 
46. Translating Research into Action TRAction. Advancing respectful maternal care and addressing disrespect \& abuse during facility-based childbirth. Bethesda: Harvard University School of Public Health (HSPH) [Internet]. 2015; Available from: www.tractionproject.org.

47. Temamo A, Abebe A, Menta A. Mothers' satisfaction with institutional delivery service and associated factors among women attending hospitals in Wolaita zone administration, SNNPR, Ethiopia. J Nutr Diet Pr [Internet]. 2018;2(2):1-12 Available from: http://www.sciaeon.org/articles/MothersSatisfaction-with-Institutional-Delivery-Service-and-Associated-Factorsamong-Women-Attending-Hospitals-in-Wolaita-Zone-Administratio.pdf.

48. Hodnett ED, Gates S, Justus Hofmeyr G, Sakala C. Continuous support for women during childbirth. Cochrane Database Syst Rev. 2014;10(25):1-59.

49. Mehata S, Paudel YR, Dariang M, Aryal KK, Paudel S, Mehta R, et al. Factors determining satisfaction among facility-based maternity clients in Nepal. BMC Pregnancy Childbirth. 2017;17(1):1-10.

50. Melese T, Gebrehiwot Y, Daniel Bisetegne DH. Assessment of client satisfaction in labor and delivery services at a maternity referral hospital in Ethiopia. Pan Afr Med J. 2014;17(76):2-9.

\section{Publisher's Note}

Springer Nature remains neutral with regard to jurisdictional claims in published maps and institutional affiliations.

Ready to submit your research? Choose BMC and benefit from:

- fast, convenient online submission

- thorough peer review by experienced researchers in your field

- rapid publication on acceptance

- support for research data, including large and complex data types

- gold Open Access which fosters wider collaboration and increased citations

- maximum visibility for your research: over $100 \mathrm{M}$ website views per year

At $\mathrm{BMC}$, research is always in progress.

Learn more biomedcentral.com/submissions 